\title{
Simulation of radiation characteristics of pulse $X$-ray devices for non-destructive testing the semiconductor materials
}

\author{
S.V. Denbnovetsky, N.V. Slobodyan \\ National Technical University "KPI”, Electronics Department, \\ 16, Politekhnichna str., 03056 Kyiv, Ukraine
}

\begin{abstract}
In the work, non-destructive testing the Si and Ge semiconductors by pulse Xray sources is discussed. Mathematical simulation of the radiation generation in reflection and transmission anode tubes is performed. Details of energy spectrum formation in these pulse tubes are analyzed, and its transformation when passing through thin samples of semiconductor materials is discussed. The dependence of the amount of radiation absorbed by the samples on the amplitude of acceleration voltage is calculated. It is shown how the pulse operation regime and design features of pulse tubes influence the characteristics of the X-ray radiation.
\end{abstract}

Keywords: non-destructive testing, pulse X-ray devices, X-ray radiation spectra, radiation absorption.

Manuscript received 02.11.05; accepted for publication 15.12.05.

\section{Introduction}

The semiconductor materials and devices (electronic industry production) as objects of the X-ray nondestructive testing have a number of specific features. It gives grounds to consider the $\mathrm{X}$-ray testing the semiconductor objects as a separate branch of investigations. The semiconductor devices are characterized by microscopic sizes of objects and expected defects, by multilayer structure of different density materials and by small absorption [1]. The vulnerability of very sensitive semiconductor device structures forces ones to limit the investigation with moderate X-ray radiation energy range and use minimal possible dose powers. The spectrum of X-ray radiation after passing the thin semiconductor structure and creating the shadow X-ray image contains almost all the low-energy components of radiation emitted by the Xray source. At the same time, the ratio of the radiation spectrum components intensities after passing through the tested semiconductor sample strongly depends on the sample thickness [2]. The necessity of taking into account the real radiation spectrum and other abovementioned factors complicates the mathematical simulation of the radiation action on the X-ray-sensitive elements of the equipment for semiconductor material investigations.

The problem of computer simulation of the process of shadow X-ray image formation by continuous operating X-ray devices for thin samples of widely used semiconductor materials $(\mathrm{Si}, \mathrm{Ge})$ was considered in [2].
There were found some features in the dependences of the X-ray radiation absorption on the acceleration voltage of an X-ray device and the sample thickness when this radiation passes through thin semiconductor samples.

However, side by side with the X-ray devices of continuous operation, pulse X-ray devices are widely used for non-destructive testing. The pulse X-ray devices have a number of advantages. First, owing to autoelectronic or explosive emission used in such devices, a system of filament heating is not required. Second, such devices can develop considerable power of $\mathrm{X}$-ray radiation during the pulse, while the average consumed power is not large. This makes a cooling system unnecessary and allows to considerably reduce the dimensions and weight of the devices [3].

The characteristics of radiation from a pulse X-ray device differ from those of a continuous operation device. The characteristics are influenced by the constant alternation of the acceleration voltage and anode current during the pulse and their periodic cessation between pulses $[4,5]$. These factors together with design features of pulse X-ray tubes considerably complicate the task to mathematically simulate generation of X-ray radiation in such sources.

In this article, we discuss the influence of the pulse operation regime on the characteristics of the X-ray radiation. We discuss the formation of the radiation spectrum and its transformation when passing through homogenous samples of different semiconductor materials. We report qualitative differences between the 
pulse and continuous regimes of operation in testing semiconductor devices and materials.

\section{Pulse X-ray tube design}

The majority of pulse X-ray tubes could be divided into tubes with a reflection anode and those with a transmission anode [4].

The reflection anode is a thin needle of a heavy metal (tungsten, molybdenum, copper etc.) 2 to $6 \mathrm{~mm}$ in diameter. It is sharpened at an angle $\alpha$ between 10 to $30^{\circ}$. In reflection anode tubes, radiation propagates in the direction of the needle axis, passes through the hollow of the cylindrical cathode and through the output beryllium window.

The transmission anode is made of tantalum or tungsten foil with the thickness $\Delta$ of 20 to $100 \mu \mathrm{m}$. In transmission anode tubes, radiation passes through the anode foil and propagates at right angle to its planar surface.

\section{Mathematical simulation}

To simulate the pulse X-ray source, it is necessary to take into account both the pulse character of operation and the design features of the specific X-ray tube.

In [6], an approximation formula for the energy spectrum of the quanta flux density for a realistic X-ray tube of continuous operation was introduced. Calculations by using this formula agree with experimental spectra to within few percents.

This model can be taken as a basis for pulse X-ray device simulation.

The device features of reflection and transmission anode tubes must be taken into account in determining the path length $y\left(E, E_{\max }\right)$ of X-ray quantum flow from where they originate in the bulk of anode to its outside surface. This path length defines the self-absorption of radiation in anode material.

The examination of the geometric scheme of generation and emission of X-ray quanta in pulse tubes of different designs enables us to express the path length as:

$y_{r}\left(E, E_{\max }\right)=\frac{\operatorname{ctg}(\alpha / 2)}{\rho_{m} c\left(E_{\max }\right)}\left(E_{\max }^{2}-E^{2}\right)$

and

$y_{t r}\left(E, E_{\max }\right)=\Delta-\frac{\left(E_{\max }^{2}-E^{2}\right)}{\rho_{m} c\left(E_{\max }\right)}$

for the tubes with reflection and transmission anodes. In these formulae, $E$ is the quantum energy in $\mathrm{keV}, E_{\max }$ is the maximal quantum energy, $\rho_{m}$ is the anode material density in $\mathrm{g} / \mathrm{cm}^{3}$, and $c\left(E_{\max }\right)$ is the ThomsonWidington constant.

To take into consideration the pulse character of the regime, the value of the maximal quantum energy $E_{\text {max }}$ (which corresponds to the accelerating anode voltage in $\mathrm{kV}$ ) and the value of the anode current $i$ must be changed to time dependent functions of these parameters (i.e., functions describing current and voltage pulse profiles) $-E_{\max }(t)$ and $i(t)$. This determines the instantaneous energy distribution of X-ray quanta at a given time point $t$. The integral of this time dependent function over the time interval of the pulse duration gives the energy distribution of X-ray quanta for one pulse. The integral of this distribution over the energy yields the total number of quanta emitted by the source per pulse.

The functions $E_{\max }(t)$ and $i(t)$ represent current and voltage pulses profiles. They depend on electrical feed circuit and physical processes in the X-ray diode. Typical shapes of anode voltage and current pulses are shown in Fig. 1a [4]. In the present work, to facilitate the calculations, we used simplified forms of pulses as shown in Fig. 1b.

The regime parameters of pulse X-ray devices are determined by the characteristics of the discharge loop, but the dependence is rather complicated. It has been shown in [4] that the dependence of the maximal value of the anode current $I_{\max }$ (in A) on the maximal quantum energy $E_{\max }$ (in $\mathrm{keV}$ ) for $\mathrm{X}$-ray tubes with explosive emission may be approximated as:

$I_{\max }\left(E_{\max }\right)=1.12 \cdot 10^{6}(C / d)^{1 / 2} E_{\max }^{3 / 4}$,

where $C$ is the condenser capacity of the pulse X-ray tube in farads; $d$ is the cathode-anode spacing in centimeters.

The averaged radiation energy distribution per pulse $N^{\sim}(E)$ for a pulse X-ray device is a sum of the two integrals over time $-D_{1}$ and $D_{2}$ divided by the pulse duration $\tau$. They correspond to two time intervals of the pulse: from 0 to $\tau_{1}$ (when the voltage is constant and the
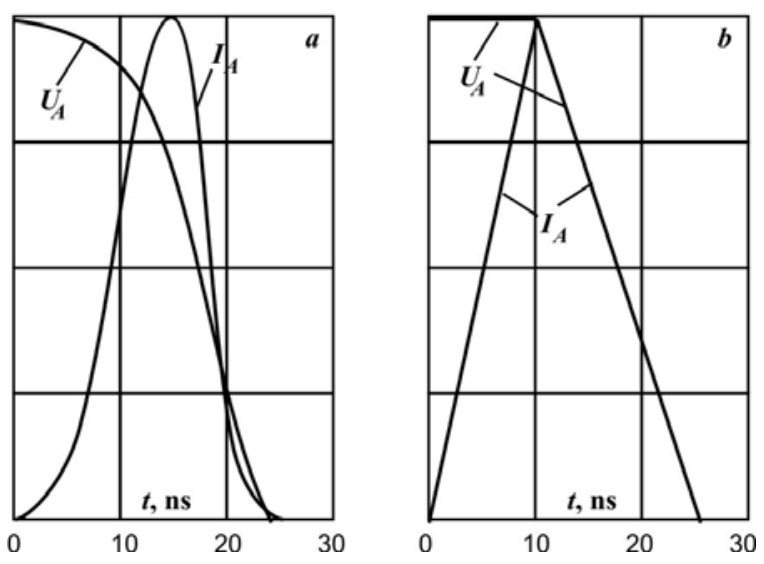

Fig. 1. Typical shapes of anode voltage and current pulses (a). Simplified forms of pulses (b). 
current linearly increases); and from $\tau_{1}$ to $\tau$ (when the voltage and the current linearly decrease to zero value). Thus,

$N^{\sim}(E)=\frac{D_{1}\left(E_{\max }, E\right)+D_{2}\left(E_{\max }, E\right)}{\tau}$.

The corresponding expressions for $D_{1}$ and $D_{2}$ are:

$D_{1}\left(E_{\max }, E\right)=\int_{0}^{\tau_{1}} N_{E}\left(E_{\max }, E\right) I\left(E_{\max }\right) \frac{t}{\tau_{1}} d t$

and

$$
\begin{aligned}
& D_{2}\left(E_{\max }, E\right)=\int_{\frac{E\left(\tau-\tau_{1}\right)}{E_{\max }}}^{\tau-\tau_{1}} N_{E}\left(E_{\max } \frac{t}{\tau-\tau_{1}}, E\right) \times \\
& \times I\left(E_{\max }\right) \frac{t}{\tau-\tau_{1}} d t .
\end{aligned}
$$

The lower limit of the integral in expression (6) is made variable to prevent the situation when the expression $\left(E_{\max }-E\right)$ becomes negative for certain relations between $t$ and $E$. In expressions (5), (6) $I\left(E_{\max }\right)$ is defined by (3); and $N_{E}\left(E_{\max }, E\right)$ is defined as:

$$
\begin{aligned}
& N_{E}\left(E_{\max }, E\right)=\frac{k_{1} Z \cdot\left(E_{\max }-E\right)}{r^{2} E} \times \\
& \times \exp \left[-\mu_{m}(E) y\left(E, E_{\max }\right)-\mu_{0}(E) H_{0}\right],
\end{aligned}
$$

where $k_{1}=1.1 \cdot 10^{12} \mathrm{~s} \cdot$ quantum $/ \mathrm{A} \cdot \mathrm{kV} ; Z$ is the atomic number of the anode material; $r$ is the distance to the tested object in centimeters; $H_{0}$ is the output X-ray tube window thickness in centimeters; $\mu_{m}(E)$ and $\mu_{0}(E)$ are the linear coefficients of X-ray radiation absorption of anode and output window materials in $\mathrm{cm}^{-1}$; the path length $y\left(E, E_{\max }\right)$ of X-ray quantum flow defined by the formulae (1) and (2) for tubes with reflection and transmission anodes, respectively. To define the pulse $\mathrm{X}$-ray radiation spectrum after passing the testing object, the expression (7) must be multiplied on $\exp [-\mu(E) H]$, where $\mu(E)$ is the linear coefficient of X-ray radiation absorption by the sample material in $\mathrm{cm}^{-1} ; H$ is its thickness in centimeters.

The dependences of the linear coefficients of X-ray radiation absorption $\mu_{m}(E), \mu_{0}(E)$ and $\mu(E)$ were obtained in this study by using cubic spline approximation of the reference-book table data of the mass coefficients of X-ray radiation absorption [7] and their multiplication on the material densities.

\section{Simulation of the spectra}

In the present calculations, typical parameters of the pulse X-ray devices "MIRA" and "ARINA" are used $[3,4]$. The parameters of pulses of the current and voltage are: $\tau=25 \mathrm{~ns}, \tau_{1}=10 \mathrm{~ns}$; the parameters in the formula (3) are: $C=40 \mathrm{pF}, d=0.2 \mathrm{~cm}$.

The normalized spectra of radiation for X-ray devices of different types are shown in Fig. 2 for comparison. These results were obtained for tungsten anodes; $E_{\max }=120 \mathrm{keV} ; r=30 \mathrm{~cm}$. In the plot, the curve 1 is the spectrum of a continuously operating device. It is calculated by the method introduced in [6]. The anode tilt angle is $19^{\circ}$; the anode current is $10 \mathrm{~mA}$; the output beryllium window thickness is $H_{0}=4 \mathrm{~mm}$. The curves 2 to 5 are the energy quantum distribution per pulse of pulse X-ray devices. They are calculated using the formula (4). The curves 2 and 3 are related to the reflection anode tube spectrum. For both tubes the thickness of output beryllium windows is $H_{0}=4 \mathrm{~mm}$, and half angles of anode sharpening are $\alpha / 2=6^{\circ}$ and $\alpha / 2=19^{\circ}$, respectively. The curves 4 and 5 are transmission anode tube spectra. The anode thickness is $\Delta=20 \mu \mathrm{m}$ and $\Delta=70 \mu \mathrm{m}$, respectively.

The analysis of the plotted relations allows us to understand the influence of pulse operation and of design features on the radiation spectrum.

To see the influence of the pulse operation regime, let us compare the curves 1 and 2 . The design dependent parameters used to calculate the curves 1 and 2 were the same: the reflection anode half-angle of the pulse tube is equal to the anode tilt angle of the continuous operating device $\left(19^{\circ}\right)$. Thus, the only difference is that in a pulse device the anode current and voltage change in time. It is seen that compared to the spectrum of the continuously operating device (curve 1) in the pulse device (curve 2) the hump is shifted to lower energies ( $~ 39 \mathrm{keV}$ and $\sim 36$ $\mathrm{keV}$, respectively). This effect agrees with experimental results for the spectrum of a pulse device $[4,8]$. The effect is explained by the fact that during a considerable part of the pulse the voltage on the pulse tube anode is lower than its amplitude value. It weakens the highenergy part of the spectrum of a single pulse. Since the low-energy part is absorbed more efficiently, the radiation absorbed by the test object must increase.

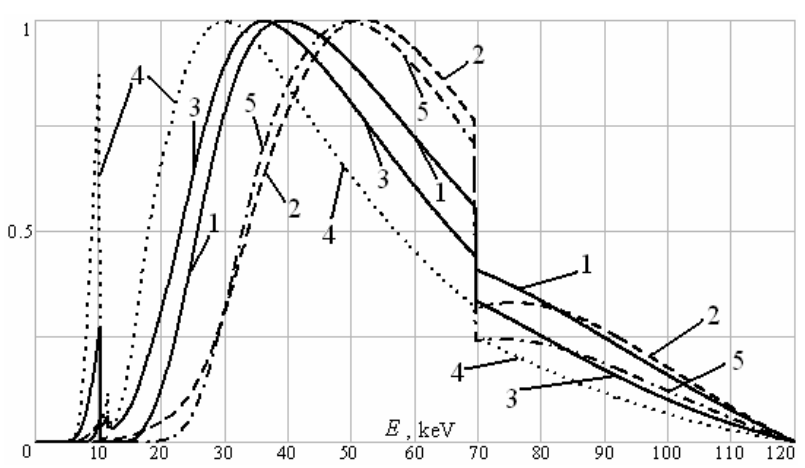

Fig. 2. The normalized spectra of radiation for X-ray devices of various types. 
To determine the influence of the design on the spectra, we performed similar calculations for a tube with the reflection anode half-angle of $\alpha / 2=6^{\circ}$ (curve 3$)$. The hump of this spectrum $(\sim 53 \mathrm{keV})$ is strongly shifted to higher energies. This is related to the fact that the distance from the point where the quanta originate to the point where they are emitted increases when the anode angle decreases (formula (1)). In this case, the design factor is stronger then the factor of the pulse operation, which acts in the opposite direction and weakens the high-energy part of the spectrum.

In the case of the transmission anode, as the foil thickness increases the hump of the spectrum shifts to higher energies: from $\sim 30 \mathrm{keV}$ (curve $4, \Delta=20 \mu \mathrm{m}$ ) to $\sim 50 \mathrm{keV}$ (curve 5; $\Delta=70 \mu \mathrm{m}$ ). When the anode is thin, the hump shifts to the left because the low-energy quanta that originate deeper in the bulk must pass a shorter distance to the outer surface then the high energy quanta. In a thick anode the pass length for quanta of all energies increases considerably and the hump in the spectrum shifts to high energies.

The simulation results are compared to the experimental spectrum of a reflection anode tube published in [8]. The characteristics of the tube were described in [9]. They correspond to the parameters of the tube, the calculated spectrum of which is shown by the curve 3 in Fig. 2. The present simulation agree with the measurements to within $12 \%$, whereas the calculation of [8] deviate from the experimental data by $44 \%$. This is because the method used in [8] does not take into account the radiation self-absorption in the target. Improved simulations introduced in these work may replace experimental investigations, which are extremely complicated in the case of pulse devices and may themselves have large errors.

\section{Pulse radiation absorption in the semiconductor samples}

As it was mentioned in the introduction, the results of simulation of the irradiation of semiconductors by the continuous X-ray radiation were presented in [2]. The tubes with tungsten and molybdenum anodes were used at a constant anode current value. The investigations show that for silicon and germanium samples of 0.03 $0.06 \mathrm{~cm}$ thickness range at certain values of anode voltage, peaks in absorption of radiation are observed. The conclusion was made that such simulations can help to determine efficient operation regimes to obtain a highquality shadow image of the defects in semiconductor structure formation.

The possibility of the efficient absorption of pulse Xray radiation in thin samples of semiconductor materials (silicon and germanium) is investigated below. The calculations were made for the parallel-sided plates of the crystalline silicon and germanium with homogenous density. The silicon and germanium sample densities used for determination of the coefficients $\mu(E)$ for
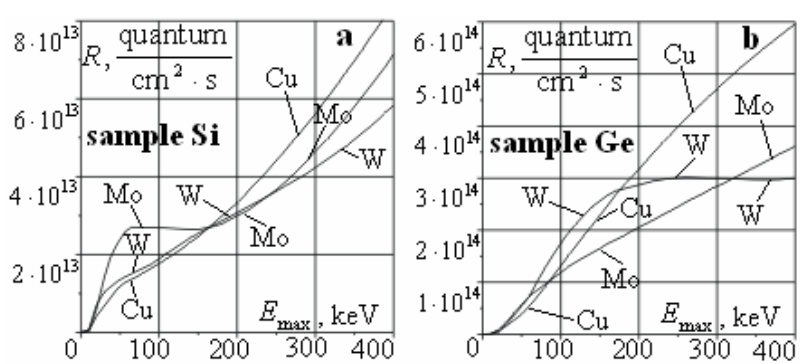

Fig. 3. The dependence of the average absorbed quantum flux per pulse on the anode voltage amplitude for the tubes with tungsten, molybdenum and copper reflection anodes: a silicon sample, $b$ - germanium sample.

these materials are 2.33 and $5.32 \mathrm{~g} / \mathrm{cm}^{3}$, respectively [10].

The results of calculations are shown in Figs 3 and 4 for the samples with $400 \mu \mathrm{m}$ thickness of silicon (Figs $3 \mathrm{a}$ and $4 \mathrm{a}$ ) and of germanium (Figs $3 \mathrm{~b}$ and $4 \mathrm{~b}$ ). Shown is the dependence of the average quantum flux absorbed by the semiconductor sample per pulse $R\left(E_{\max }\right)$ on the anode voltage amplitude. For the tubes with tungsten, molybdenum and copper reflection anodes corresponding dependences are shown in Fig. 3. (The anode half-angle was $\left.\alpha / 2=6^{\circ}\right)$. For the tubes with a tungsten transmission anode the results are shown in Fig. 4. (The anode thicknesses were 20, 30, 50, 70, $100 \mu \mathrm{m})$.

It can be seen that in contrast to continuously operating devices [2], for pulse X-ray sources and thin semiconductor samples there are no clear absorption maxima. Nevertheless, for the reflection anode tube in the case of silicon sample (Fig. 3a) for the Mo target the curve saturates (from 60 to $160 \mathrm{keV}$ ) and there is the evident knee in the neighborhood of $50 \mathrm{keV}$ for the $\mathrm{W}$ and $\mathrm{Cu}$ targets. In the case of germanium sample, there is a peak of absorption at $E_{\text {max }}=260 \mathrm{keV}$ for the tungsten target (Fig. 3b).

For transmission anode tubes, the absorbed radiation part increases with the amplitude of anode voltage, and

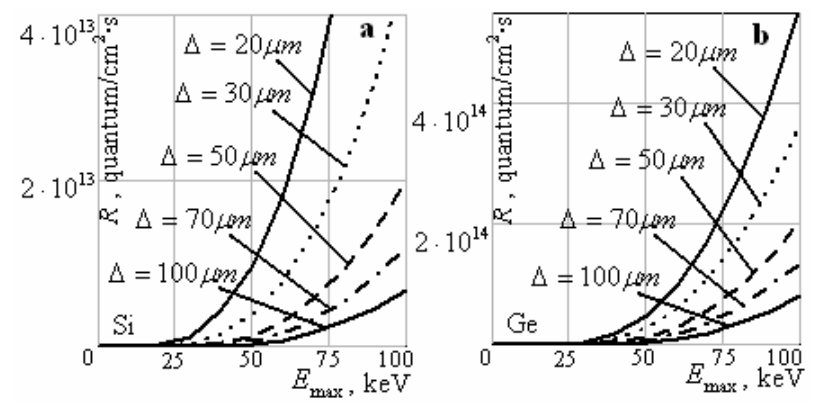

Fig. 4. The dependence of the average absorbed quantum flux per pulse on the anode voltage amplitude for the tube with a tungsten transmission anode: $\mathrm{a}$ - silicon sample, $\mathrm{b}-$ germanium sample. 
there are no singularities in the corresponding plots for the samples of silicon and germanium (Fig. 4). The thinner the target, the steeper the curves.

\section{Conclusions}

1. The developed mathematical simulation of the radiation characteristics of pulse X-ray devices agrees with experimental results.

2. The pulse regime of operation and design features of the pulse X-ray device (the electrode configuration and the discharge gap geometry) strongly influence the energy distribution of X-ray quanta.

3 . The pulse regime of operation leads to a shift of the spectrum hump to lower energies compared to the spectrum of a continuously operating device.

4. In pulse X-ray devices, a decrease of the reflection anode angle or an increase of the thickness of a transmission anode lead to a shift of the energy spectrum hump to higher energies.

5. In pulse X-ray devices with reflection anode tubes, the anode voltage dependence of the radiation absorption in thin semiconductor samples has under certain conditions some structures, but they are not as pronounced as those in continuously operating devices.

6. For transmission anode tubes, the absorption in silicon and germanium steadily increases with the pulse voltage amplitude.

\section{References}

1. The non-destructive testing of the elements and the units of the electronics / Ed. by B.E. Berdichevsky. Sovetskoe radio, Moscow (1976) (in Russian).
2. S.V. Denbnovetsky, A.V. Leshchishin, N.V. Slobodyan, The X-ray radiation absorption in silicon and germanium // Elektronika i svyaz' No 20, p. 109112 (2003) (in Russian).

3. The X-ray technique. Reference book: In 2 books. Book 1 / Ed. by V.V. Cluev. Mashinostroenie, Moscow (1980) (in Russian).

4. S.P. Vavilov, V.I. Gorbunov, The pulse X-ray radiation in defectoscopy. Energoatomizdat, Moscow (1985) (in Russian).

5. The X-ray devices radiation spectra: Reference book // V.N. Vasiliev, L.A. Lebedev, V.P. Sidorin, R.V. Stavitskii. Energoatomizdat, Moscow (1990) (in Russian).

6. S.R. Mikhailov, The simulation of the shadow X-ray testing object image in roentgenoscopy systems for non-destructive testing // Elektronika i svyaz' No 16, p. 59-70 (2002) (in Russian).

7. Storm Ellery, Israel Harvey, Photon cross sections from 0.001 to $100 \mathrm{meV}$ for elements 1 through 100 . Los Alamos Scientific Laboratory, New Mexico (1967).

8. U.M. Tolchenov, A.V. Chepeck, The pulse X-ray tube with cold cathode radiation spectrum // Pribory i tekhnika eksperimenta No 4, p. 233-235 (1972) (in Russian).

9. L.S. Eig, V.N. Dremin, V.V. Latyshev, The pulse $\mathrm{X}$-ray regulated tubes with cold cathode // Pribory $i$ texnika eksperimenta No 4, p. 180-182 (1969) (in Russian).

10. The materials of instrument-making industry and automatics. Reference book / Ed. by U.M. Pyatin. Mashinostroenie, Moscow (1969) (in Russian). 